\title{
Theory of Martensitic Phase Transitions in Elastoplastic Materials
}

\author{
V.I. Levitas \\ Institut für Baumechanik und Numerische Mechanik, Universität Hannover, Appelstrasse 9A, \\ 30167 Hannover, Germany
}

\begin{abstract}
The conditions of nucleation, nondisappearance of nucleus and interface propagation are derived for noncoherent and coherent phase transitions (PT) in elastoplastic materials. Extremum principles for determination of all unknown parameters are derived based on the postulate of realizability. Two model problems are solved. The predicted effect of shear stresses and plastic strains on PT pressure and hysteresis is in qualitative agreement with experiments.
\end{abstract}

\section{Introduction}

For the description of PT in elastic materials the principle of the minimum of free energy is usually used. But for media with dissipation the corresponding principle is lacking. For elastic materials with hysteresis and elastoplastic materials PT criterion was suggested (see [1]-[3] and references) in the following form: the driving force of PT reaches some threshold value. In $[4,5]$ it was obtained that for elastoplastic materials an additional threshold, related with the plastic dissipation, should be introduced and can be easily calculated. But PT criterion is only one scalar equation, which is not sufficient for the determination of all parameters, e.g. shape and orientation of nuclei, jump of strain, etc. For these purposes in $[2,4,5]$ some extremum principles were substantiated, which were also applied for the noncoherent PT. The present paper is a further development of the methods suggested in $[1,2,4]$. Both coherent (jump of the position vector $\mathbf{r}$ across the interface $[\mathbf{r}]:=\mathbf{r}_{2}-\mathbf{r}_{1}=0$, where subscripts 1 and 2 denote the phase before and after $\mathrm{PT})$ and noncoherent $([\mathbf{r}] \neq 0) \mathrm{PT}$ are considered; but $[r]$ is geometrically small in the sense that the same points of the interface remain in contact.

\section{Conditions of nucleation, nondisappearance of nucleus and interface propagation}

Consider a volume $V$ of austenite + martensite mixture with a boundary $S$. Assume that on part of surface $S$ the stress vector $\mathbf{p}$ is prescribed and on the rest part the velocity vector $\mathbf{v}$ is given, but a mixed boundary conditions (BC) are also possible. For the isothermal processes the dissipation increment $N$ during the time $\Delta t$ can be defined, using the second law of thermodynamics $[4,5]$

$$
N:=\int_{t}^{t+\Delta t} \int_{S} \mathrm{p} \cdot \mathrm{v} d S d t-\int_{t}^{t+\Delta t} \frac{d}{d t} \int_{V} \psi d V d t \geq 0
$$

where $\psi$ is the Helmholtz free energy per unit volume. Let us divide the volume $V$ into three parts: 
the volume $V_{n}$ with the boundary $\Sigma_{n}$, in which due to $\mathrm{PT}$ during the time $\Delta t$ the new nuclei appeared; the volume $\int_{\Sigma} v_{n} d \Sigma \Delta t$ covered by the moving interface $\Sigma$ with normal velocity $v_{n}$; the rest volume $V_{1}$ in which $\mathrm{PT}$ do not occur during the time $\Delta t$. Using the Gauss theorem for the volume $V$ with the discontinuity surfaces $\Sigma+\Sigma_{n}$ and the formula for differentiating on a variable volume we obtain a decomposition of $N$ into dissipation increments in each of the above volumes

$$
\begin{aligned}
& N=N_{1}+N_{n}+\int_{t}^{t+\Delta t} \int_{\Sigma+\Sigma_{n}} \tilde{X}_{\Sigma} d \Sigma d t, \quad N_{1}=\int_{t}^{t+\Delta t} \int_{V_{1}}(\boldsymbol{\sigma}: \dot{\varepsilon}-\dot{\psi}) d t d V_{1} \\
& N_{n}=\int_{\varepsilon_{1}}^{\varepsilon_{2}} \int_{V_{n}} \boldsymbol{\sigma}: d \varepsilon d V_{n}-\int_{V_{n}}\left(\psi_{2}-\psi_{1}\right) d V_{n}, \quad \tilde{X}_{\Sigma}=-\mathrm{p} \cdot[\mathrm{v}]-[\psi] v_{n}
\end{aligned}
$$

where $\boldsymbol{\sigma}$ and $\boldsymbol{\varepsilon}$ denote the stress and the strain tensors, and we took into account $[\mathbf{p}]=0$. Due to the mutual independence of each of three integrals in Eq. (2), each of them is nonnegative.

For elastic materials without dissipation conditions $N_{n}=0$ and $\tilde{X}_{\Sigma}=0 \quad \forall \mathrm{r} \in \Sigma$ can be considered as equilibrium nucleation and interface propagation conditions, because they describe PT at zero dissipation. For PT in elastoplastic materials and in elastic materials with the hysteresis these conditions can not be applied, because the finite dissipation occurs in course of PT.

Consider the volume $V_{n}$ and assume that we can determine the actual value of the dissipation increment $N_{n}^{a}$ in course of PT in $V_{n}$ and $N_{n}^{a}=\int\left(k_{n}^{e}+k_{n}^{p}\right) d V_{n}$, where $k_{n}^{p}=\int\left(\sigma-\frac{\partial \psi}{\partial \varepsilon^{p}}\right): d \varepsilon^{p}$ and $k_{n}^{e}$ are the plastic and "nonplastic" dissipation increment in a unit volume, $\varepsilon^{p}$ the plastic strain. The term $k_{n}^{e}$ is related with the emission of acoustic waves, lattice friction and it is responsible for the hysteresis at PT in elastic materials [1]-[6].

Both $N_{n}$ and $N_{n}^{a}$ depend on the BC. If at given BC $N_{n}<N_{n}^{a}$, then PT will not take place, because for actually occurring PT $N_{n}=N_{n}^{a}$, as $N_{n}^{a}$ is by definition the actual value of dissipation increment. Consequently, the necessary condition of nucleation is $N_{n}=N_{n}^{a}$, i.e.

$$
\begin{gathered}
\int_{\varepsilon_{1}}^{\varepsilon_{2}} \int_{V_{n}} \sigma: d \varepsilon d V_{n}-\int_{V_{n}}\left(\psi_{2}-\psi_{1}\right) d V_{n}=\int_{V_{n}}\left(k_{n}^{e}+k_{n}^{p}\right) d V_{n}>0 \\
\text { or } \quad \int_{t}^{t+\Delta t} \int_{\Sigma_{n}} \mathbf{p} \cdot \mathbf{v} d \Sigma_{n} d t-\int_{V_{n}}\left(\psi_{2}-\psi_{1}\right) d V_{n}=\int_{V_{n}}\left(k_{n}^{e}+k_{n}^{p}\right) d V_{n}>0,
\end{gathered}
$$

where $\mathrm{v}$ is the velocity on $\Sigma_{n}$ from the side of nucleus. Note that Gauss theorem was used. If we decompose $\varepsilon=\varepsilon^{e}+\varepsilon^{p}+\varepsilon^{f}$, where $\varepsilon^{e}$ and $\varepsilon^{f}$ are elastic and transformation strains, then terms $\sigma: d \varepsilon^{p}$ in the left and right parts of Eq. (3) eliminate each other and we obtain

$$
\int_{\varepsilon_{1}}^{\varepsilon_{2}} \int_{V_{n}}\left(\sigma: d\left(\varepsilon^{e}+\varepsilon^{f}\right)+\frac{\partial \psi}{\partial \varepsilon^{p}}: d \varepsilon^{p}\right) d V_{n}-\int_{V_{n}}\left(\psi_{2}-\psi_{1}\right) d V_{n}-\int k_{n}^{e} d V_{n}=\mathbf{0}
$$

At $\frac{\partial \psi}{\partial \varepsilon^{p}}=0 \mathrm{Eq}$. (5) has the same form as for PT in elastic materials; plasticity effects on a variation of $\boldsymbol{\sigma}$ in the course of PT and on the value $k_{n}^{e}$. If $\psi_{i}=1 / 2 \varepsilon_{i}^{e}: \mathbf{E}_{i}: \varepsilon_{i}^{e}+\psi_{i}^{\theta}, i=1,2$ and $\mathbf{E}_{1}=\mathbf{E}_{2}$, where $\mathbf{E}_{i}$ are the tensors of elastic moduli of $i$-phase, $\psi_{i}^{\theta}$ the thermal part of the free energy, then

$$
\int_{\varepsilon_{1}^{f}}^{\varepsilon_{2}^{f}} \int_{V_{n}} \boldsymbol{\sigma}: d \mathrm{e}^{f} d V_{n}-\int_{V_{n}}\left(\psi_{2}^{\theta}-\psi_{1}^{\theta}\right) d V_{n}-\int_{V_{n}} k_{n}^{e} d V_{n}=0
$$

i.e. the elastic strains also disappear. A similar consideration gives a condition of interface propagation

$$
\tilde{X}_{\Sigma}=-\mathbf{p} \cdot[\mathbf{v}]-[\psi] v_{n}=\tilde{k}_{1 \rightarrow 2}^{e}+\tilde{k}_{1 \rightarrow 2}^{p}>0 \quad \forall \mathbf{r} \in \Sigma, \quad t^{\prime} \in[t, t+\Delta t]
$$


where $\tilde{k}_{1 \rightarrow 2}^{p}$ and $\tilde{k}_{1 \rightarrow 2}^{e}$ are the actual value of the dissipation rate due to plastic deformation and nonplastic effect (e.g. due to intersection of interface with different types of defects and lattice friction) at the moving interface. For coherent PT due to compatibility condition $[2,4][\mathbf{F}]=$ $=-[\mathbf{v}] \mathbf{n} / v_{n}$, whence $[\mathbf{v}]=-[\mathbf{F}] \cdot \mathbf{n} v_{n}, \quad[\mathbf{F}]=[\mathbf{F}] \cdot \mathbf{n} \mathbf{n}$, where $\mathbf{F}=\boldsymbol{\varepsilon}+\boldsymbol{\omega}, \quad \boldsymbol{\omega}$ is the rotation tensor, $\mathbf{n}$ the unit normal to the interface. We obtain $-\mathbf{p} \cdot[\mathbf{v}]=\mathbf{n} \cdot \boldsymbol{\sigma} \cdot[\mathbf{F}] \cdot \mathbf{n} v_{n}=\boldsymbol{\sigma}:([\mathbf{F}] \cdot \mathbf{n} \mathbf{n}) v_{n}=$ $=\sigma:[\mathbf{F}] v_{n}=\sigma:[\varepsilon] v_{n}$. Introducing $X_{\Sigma}=\tilde{X}_{\Sigma} / v_{n}, k_{1 \rightarrow 2}^{e}=\tilde{k}_{1 \rightarrow 2}^{e} / v_{n}$ and $k_{1 \rightarrow 2}^{p}=\tilde{k}_{1 \rightarrow 2}^{p} / v_{n}$ from Eq. (7) we have

$$
\begin{gathered}
X_{\Sigma}=\sigma:[\varepsilon]-[\psi]=k_{1 \rightarrow 2}^{e}+k_{1 \rightarrow 2}^{p} \quad \forall \mathbf{r} \in \Sigma, \quad t^{\prime} \in[t, t+\Delta t] \\
\text { As } \quad k_{1 \rightarrow 2}^{p}=\int_{\varepsilon_{1}}^{\varepsilon_{2}} \boldsymbol{\sigma}: d \varepsilon-\left(\psi_{2}\left(\varepsilon_{2}^{e}, \varepsilon_{2}^{p}\right)-\psi_{1}\left(\varepsilon_{1}^{e}, \varepsilon_{1}^{p}\right)\right)
\end{gathered}
$$

then substituting of Eq. (9) into Eq. (8) we obtain the generalized Maxwell rule $\left(\dot{u}_{n}=v_{n}\right)$

$$
\boldsymbol{\sigma}:[\varepsilon]=\int_{\varepsilon_{1}}^{\varepsilon_{2}} \boldsymbol{\sigma}: d \varepsilon+k_{1 \rightarrow 2}^{e}\left(u_{n}\right), \quad t^{\prime} \in[t, t+\Delta t] .
$$

Let write Eq. (10) at time $t+\Delta t$ (subscript $\Delta$ denotes that parameter is determined at time $t+\Delta t$ )

$$
\sigma_{\Delta}:\left(\varepsilon_{2 \Delta}-\varepsilon_{1 \Delta}\right)=\int_{\varepsilon_{1 \Delta}}^{\varepsilon_{2 \Delta}} \sigma: d \varepsilon+k_{1 \rightarrow 2}^{e}\left(u_{n \Delta}\right) .
$$

For an infinitesimal $\Delta t$ Eqs. (11) and (10) at $t^{\prime}=t$ can be transformed into

$$
\left(\dot{\boldsymbol{\sigma}}+\mathbf{n} \cdot \nabla \boldsymbol{\sigma} v_{n}\right):\left(\varepsilon_{2}-\varepsilon_{\mathbf{1}}\right)=\frac{\partial k_{1 \rightarrow 2}^{e}}{\partial u_{n}} v_{n}
$$

We have taken into account that $\boldsymbol{a}_{\Delta}=\boldsymbol{a}+\left(\dot{\boldsymbol{a}}+\mathbf{n} \cdot \nabla \boldsymbol{a} v_{n}\right) \Delta t$, for $\boldsymbol{a}=\boldsymbol{\sigma}, \boldsymbol{\varepsilon}_{2}$ and $\boldsymbol{\varepsilon}_{1}, \boldsymbol{\nabla}$ is the gradient operator, where the term $\nabla a$ appears due to the fact that the tensor $a_{\Delta}$ is determined on the $\Sigma_{\Delta}$ surface, i.e. at point $\mathbf{r}+v_{n} \mathbf{n} \Delta t(\mathbf{r} \in \Sigma(t))$. Consequently, Eqs. (10) at $t^{\prime}=t$ and (12) are the necessary conditions for the coherent interface propagation.

It is not necessary that Eqs. (10) and (11) are valid for the points of $\Sigma_{n}$, because after nucleation the interface $\Sigma_{n}$ can be fixed. But we should be sure that $\Sigma_{n}$ does not move back and nucleus does not disappear. The condition of nondisappearance of nucleus is a violation of the propagation condition, when $v_{n}<0$ and PT $2 \rightarrow 1$ occurs, i.e.

$$
\tilde{X}_{\Sigma}=-\mathbf{p} \cdot[\mathbf{v}]-[\psi] v_{n}<\tilde{k}_{2 \rightarrow 1}^{e}+\tilde{k}_{2 \rightarrow 1}^{p} \quad \forall \mathbf{r} \in \Sigma_{n}, \quad t^{\prime} \in[t, t+\Delta t]
$$

or for coherent $\mathrm{PT}$, introducing $k_{2 \rightarrow 1}^{e}=-\tilde{k}_{2 \rightarrow 1}^{e} / v_{n}>0, \quad k_{2 \rightarrow 1}^{p}=-\tilde{k}_{2 \rightarrow 1}^{p} / v_{n}>0$,

$$
X_{\Sigma}=\boldsymbol{\sigma}:[\varepsilon]-[\psi]>-k_{2 \rightarrow 1}^{e}-k_{2 \rightarrow 1}^{p}<0
$$

\section{Model problems}

To receive a feeling how plastic strain and noncoherency effect on PT let us consider two simple plane strain problems. Assume that the materials are perfectly rigid-plastic.

1. Consider an infinite half-space with prescribed normal $\sigma_{n}$ and shear $\tau$ stresses on the whole surface (Fig. 1). Assume that a coherent PT occurs in the layer along the whole surface and the solution does not depend on the $x$ coordinate. The same solution is valid, if PT occurs in the parallel to the external surface layer inside of the half-space. Material outside of the layer is rigid. 
Let $\varepsilon^{f}=1 / 2 \varepsilon_{0} \mathbf{I}+\gamma^{f}(\mathbf{t n})_{s}$, where $\varepsilon_{0}$ and $\gamma^{f}$ are the volumetric and shear transformation strains, $\mathbf{I}$ the two dimensional unit tensor, subscript $s$ means symmetrization. The von Mises yield condition results in $\left(\sigma_{n}-\sigma_{t}\right)^{2}+4 \tau^{2}=\sigma_{y}^{2}$ [2], where $\sigma_{t}$ is the tangential stress, $\sigma_{y}$ the yield limit at simple compression (tension) of phase 2 .

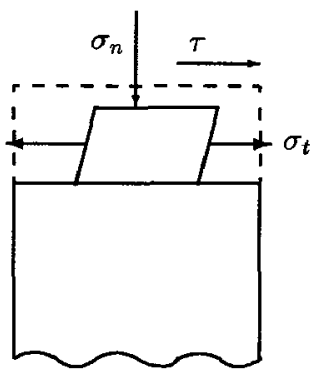

a

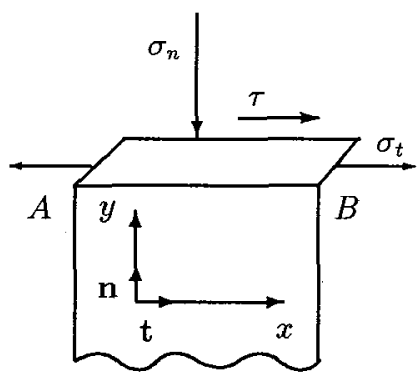

$\mathrm{b}$

Fig. 1. Coherent PT in thin layer

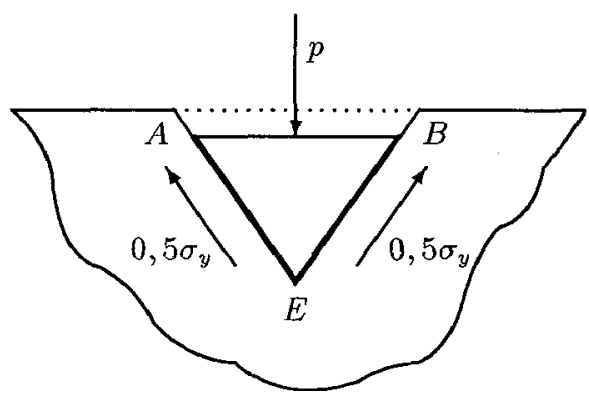

Fig. 2. Noncoherent PT under local pressure.

In Fig. 1a transformed particle is shown after transformation strain, but to satisfy displacement continuity across the interface $A B$ and independence of solution of $x$, additional plastic strain is needed (Fig. 1b). Nucleation criterion (6) gives

$$
X_{n}=1 / 2\left(\sigma_{n} \varepsilon_{0}+\sigma_{t} \varepsilon_{0}\right)+\tau \gamma^{f}-\Delta \psi^{\theta}=k_{n, \mathbf{1} \rightarrow 2}^{e} .
$$

From the yield condition $\sigma_{t}=\sigma_{n}-\sqrt{\sigma_{y}^{2}-4 \tau^{2}}$. Substituting $\sigma_{t}$ in Eq. (15) we obtain PT pressure

$$
\sigma_{n}^{1 \rightarrow 2}=\frac{\Delta \psi^{\theta}}{\varepsilon_{0}}+\frac{k_{n, 1 \rightarrow 2}^{e}}{\varepsilon_{0}}+\frac{\sqrt{\sigma_{y}^{2}-4 \tau^{2}}}{2}-\frac{\tau \gamma^{f}}{\varepsilon_{0}} .
$$

For reverse PT we have $X_{n}=-1 / 2\left(\sigma_{n} \varepsilon_{0}+\sigma_{t} \varepsilon_{0}\right)-\tau \gamma^{f}+\Delta \psi^{\theta}=k_{n, 2 \rightarrow 1}^{e}>0$. From the yield condition in this case $\sigma_{t}=\sigma_{n}+\sqrt{\sigma_{y}^{2}-4 \tau^{2}}$, because plastic compression in direction $\mathbf{t}$ is possible at $\sigma_{t}>\sigma_{n}$ only. Substituting $\sigma_{t}$ in the expression for $X_{n}$ we obtain the reverse PT pressure

$$
\sigma_{n}^{2 \rightarrow 1}=\frac{\Delta \psi^{\theta}}{\varepsilon_{0}}-\frac{k_{n, 2 \rightarrow 1}^{e}}{\varepsilon_{0}}-\frac{\sqrt{\sigma_{y}^{2}-4 \tau^{2}}}{2}-\frac{\tau \gamma^{f}}{\varepsilon_{0}} .
$$

The pressure hysteresis is $H=\sigma_{n}^{1 \rightarrow 2}-\sigma_{n}^{2 \rightarrow 1}=\sqrt{\sigma_{y}^{2}-4 \tau^{2}}+\left(k_{n, 1 \rightarrow 2}^{e}+k_{n, 2 \rightarrow 1}^{e}\right) / \varepsilon_{0}$. Let us analyze the result. At $\gamma^{f}=0$, despite the fact that $\tau$ does not contribute to $X_{n}$, due to appearance of $\tau$ in the yield condition, it can significantly reduce $\sigma_{n}^{1 \rightarrow 2}$ and $H$, which is in agreement with experiments [7]. At $\tau=\tau_{\max }=1 / 2 \sigma_{y}$ the terms with $\sigma_{y}$ disappear in the expressions for $\sigma_{n}$ and $H$. At $\gamma^{f} \neq 0 \quad \sigma_{n}^{1 \rightarrow 2}$ receives an additional reduction and at large $\tau$ and $\gamma^{f}$ it is possible that $\sigma_{n}^{1 \rightarrow 2}<\Delta \psi^{\theta} / \varepsilon_{0}$. This result is in agreement with experiments [7], in which applied $\tau$ or shear plastic strain reduce the PT pressure up to a value, which is less than thermodynamical equilibrium $\sigma_{n}=\Delta \psi^{\theta} / \varepsilon_{0}$. Note that the associated flow rule reads

$$
\dot{\varepsilon}^{p}=h\left(\sigma_{n}-\sigma_{t}\right) \quad \dot{\gamma}^{p}=4 h \tau, \quad \dot{\gamma}^{p} / \dot{\varepsilon}^{p}=4 \tau /\left(\sigma_{n}-\sigma_{t}\right),
$$

where $\varepsilon^{p}$ and $\gamma^{p}$ are the normal (along $\mathbf{n}$ ) and the shear plastic strain, $h$ a scalar. From the plastic incompressibility and the condition $\varepsilon_{t}=0$ (Fig.1) it follows $\varepsilon^{p}=1 / 2 \varepsilon_{0}$. Then from Eq. (18)

$$
\gamma^{p}=\varepsilon_{0} \frac{a}{\sqrt{1-a^{2}}}, \quad a=\frac{2 \tau}{\sigma_{y}}, \quad \frac{\sqrt{\sigma_{y}^{2}-4 \tau^{2}}}{2}=\frac{\sigma_{y}}{2} \frac{\varepsilon_{0}^{2}}{\varepsilon_{0}^{2}+\gamma^{p 2}}, \quad \text { whence }
$$




$$
\sigma_{n}^{1 \rightarrow 2}=\frac{\Delta \psi^{\theta}}{\varepsilon_{0}}+\frac{k_{n, 1 \rightarrow 2}^{e}}{\varepsilon_{0}}+\frac{\sigma_{y}}{2} \frac{\varepsilon_{0}^{2}}{\varepsilon_{0}^{2}+\gamma^{p 2}}-\frac{\tau \gamma^{f}}{\varepsilon_{0}} .
$$

Eq. (19) exhibits the experimentally observed [7] decrease of PT pressure under the growing shear plastic strain; at $2 \tau=\sigma_{y} \quad \gamma^{p} \rightarrow \infty$ and the term with $\sigma_{y}$ in Eq. (19) disappears. If on the surface $S$ the horizontal displacement $u$ instead of $\tau$ is prescribed, then $\gamma^{p}=u / b$, where $b$ is the width of transformed layer. Minimal value $\sigma_{n}^{1 \rightarrow 2}$ will be at $b \rightarrow 0$ and $\gamma^{p} \rightarrow \infty$, i.e. PT starts in infinitesimal narrow layer, i.e. on the slip surface, which is in agreement with experiments.

Let us consider conditions (8) and (14) for the interface $\Sigma$. In our case $[\varepsilon]=\varepsilon_{0} \mathbf{n} \mathbf{n}+\left(\gamma^{f}+\gamma^{p}\right)(\mathbf{t n})_{s}, k_{1 \rightarrow 2}^{p}=1 / 2\left(\sigma_{n}-\sigma_{t}\right) \varepsilon_{0}+\tau \gamma^{p}$ and Eq. (8) results in Eq. (15) with $k_{1 \rightarrow 2}^{e}$ instead of $k_{n, 1 \rightarrow 2}^{e}$. Eq. (14) results in $1 / 2\left(\sigma_{n} \varepsilon_{0}+\sigma_{t} \varepsilon_{0}\right)+\tau \gamma^{f}-\Delta \psi^{\theta}>-k_{2 \rightarrow 1}^{e}<0$ and condition of nondisappearance of nucleus is met automatically, because at nucleation the right side of previous equation is equal to $k_{1 \rightarrow 2}^{e}>0$. As driving forces for the nucleation and the interface propagation are the same (Eq. (15)), further nucleation places and growth of nuclei depend on functions $k_{n}^{e}(y)$ and $k_{1 \rightarrow 2}^{e}\left(y, u_{n}\right)$. This problem will be considered elsewhere.

2. Let under the action of homogeneously distributed normal stress $p$ over the length $a \mathrm{PT}$ occurs in triangle $A B E$ (sides $A E$ and $B E$ are orthogonal), the rest part of the half-space is rigid. The transformation strain $\varepsilon^{f}=1 / 2 \varepsilon_{0} \mathbf{I}, \quad \varepsilon^{p}=0$ in the triangle $A B E$, where $\varepsilon_{0}=1-V_{n 2} / V_{n 1}$. Along the interfaces $A E$ and $B E \quad[\mathbf{r}] \neq 0$, i,e. noncoherent PT occurs. Assume that the shear stress along the interfaces $A E$ and $B E$ is maximal, $\tau=1 / 2 \sigma_{y}$, where $\sigma_{y}$ is the yield limit of weaker material. To avoid plastic strain in the triangle $A B E$ we should assume that the new phase is stronger. From the plastic limit equilibrium theory we obtain that at $p<(1+\pi / 2) \sigma_{y}$ the half-space is rigid. The nucleation condition (4) results in

$$
\int_{t}^{t+\Delta t} \int_{\Sigma_{n}} \mathbf{p} \cdot \mathbf{v} d \Sigma_{n} d t=\left(\Delta \psi^{\theta}+k_{n, 1 \rightarrow 2}^{e}\right) V_{n 1} .
$$

In the given problem we can consider also a large $\varepsilon_{0}$, if we assume that the values $\Delta \psi^{\theta}$ and $k_{n}$ are referred to the unit volume of phase 1 . With taking into account the variation in geometry in the course of PT for work $A_{p}$ of $p$ stress and work $A_{\tau}$ of shear stresses along lines $A E$ and $B E$ we obtain $A_{p}=-p\left(h_{2}^{2}-h_{1}^{2}\right)=p \varepsilon_{0} V_{n 1} \quad(p>0), \quad A_{\tau}=-1 / 2 \sigma_{y}\left(h_{1}-h_{2}\right)^{2}=-1 / 2 \sigma_{y}\left(1-\sqrt{1-\varepsilon_{0}}\right)^{2} V_{n 1}$, where $h_{1}$ and $h_{2}$ are the initial and final vertical heights of the triangle $A B E, V_{n 1}=h_{1}^{2}$, $\varepsilon_{0}=1-\left(h_{2} / h_{1}\right)^{2}$. As left side of Eq. (20) is equal to $A_{p}+A_{\tau}$, than we have

$$
p_{1 \rightarrow 2}=\frac{1}{\varepsilon_{0}}\left[1 / 2 \sigma_{y}\left(1-\sqrt{1-\varepsilon_{0}}\right)^{2}+\Delta \psi^{\theta}+k_{n, 1 \rightarrow 2}^{e}\right] \text {. }
$$

Similar calculations give $p_{2 \rightarrow 1}=\left[-1 / 2 \sigma_{y}\left(1-\sqrt{1-\varepsilon_{0}}\right)^{2}+\Delta \psi^{\theta}-k_{n, 2 \rightarrow 1}^{e}\right] / \varepsilon_{0}$, $H=p_{1 \rightarrow 2}-p_{2 \rightarrow 1}=\left[\sigma_{y}\left(1-\sqrt{1-\varepsilon_{0}}\right)^{2}+\left(k_{n, 1 \rightarrow 2}^{e}+k_{n, 2 \rightarrow 1}^{e}\right)\right] / \varepsilon_{0}$. At $\varepsilon_{0} \ll 1$ the coefficient $B:=\varepsilon_{0}^{-1}\left(1-\sqrt{1-\varepsilon_{0}}\right)^{2} \simeq 0,25$ and $B$ decreases when $\varepsilon_{0}$ growth. Consequently, the contribution of the dissipation due to noncoherency to $p_{1 \rightarrow 2}$ and $H$ is smaller, than the contribution of the dissipation due to plastic flow to $\sigma_{n}^{1 \rightarrow 2}$ and $H$ in the previous problem at $\tau=0$.

\section{The postulate of realizability}

To determine all unknown parameters $\mathbf{b}$ (position, shape and orientation of nucleus, $\varepsilon^{f}, \varepsilon_{2}$ and $\varepsilon_{1}$ and so on) let us use the postulate of realizability [4, 5]:

If starting from the state with $N_{n}\left(\mathbf{b}^{*}\right)<N_{n}^{a}\left(\mathbf{b}^{*}\right)$ (or $\left.\tilde{X}_{\Sigma}\left(\mathbf{b}^{*}\right)<\tilde{k}^{e}\left(\mathbf{b}^{*}\right)+\tilde{k}^{p}\left(\mathbf{b}^{*}\right)\right)$ for all possible PT parameters $b^{*}$ (i.e. PT does not occur) in the course of variation of $\mathrm{BC}$ the condition 
$N_{n}(\mathbf{b})=N_{n}^{a}(\mathbf{b})\left(\right.$ or $\left.\tilde{X}_{\Sigma}(\mathbf{b})=\tilde{k}^{e}(\mathbf{b})+\tilde{k}^{p}(\mathbf{b})\right)$ is fulfilled the first time for some of parameters $\mathbf{b}$, then nucleation (or interface propagation) will occur with this $\mathbf{b}$ (if condition $\tilde{X}_{\Sigma}(\mathbf{b})=\tilde{k}^{e}(\mathbf{b})+$ $\tilde{k}^{p}(\mathbf{b})$ is not violated in the course of interface propagation).

If, in the course of variation of $\mathrm{BC}$ condition $N_{n}(\mathbf{b})=N_{n}^{a}(\mathbf{b})\left(\right.$ or $\left.\tilde{X}_{\Sigma}(\mathbf{b})=\tilde{k}^{e}(\mathbf{b})+\tilde{k}^{p}(\mathbf{b})\right)$ is met for one or several $\mathbf{b}$, then for arbitrary other $\mathbf{b}^{*}$ inequality $N_{n}\left(\mathbf{b}^{*}\right)<N_{n}^{a}\left(\mathbf{b}^{*}\right)$ (or $\tilde{X}_{\Sigma}\left(\mathbf{b}^{*}\right)<$ $<\tilde{k}^{e}\left(\mathbf{b}^{*}\right)+\tilde{k}^{p}\left(\mathbf{b}^{*}\right)$ ) should be held, as in the opposite case for this $\mathbf{b}^{*}$ condition $N_{n}=N_{n}^{a}$ (or $\left.\tilde{X}_{\Sigma}=\tilde{k}^{e}+\tilde{k}^{p}\right)$ had to be met before it was satisfied for $\mathbf{b}$. Consequently, we obtain the extremum principles

$$
\begin{gathered}
N_{n}\left(\mathbf{b}^{*}\right)-N_{n}^{a}\left(\mathbf{b}^{*}\right)<0=N_{n}(\mathbf{b})-N_{n}^{a}(\mathbf{b}) \\
\tilde{X}_{\Sigma}\left(\mathbf{b}^{*}\right)-\tilde{k}^{e}\left(\mathbf{b}^{*}\right)-\tilde{k}^{p}\left(\mathbf{b}^{*}\right)<0=\tilde{X}_{\Sigma}(\mathbf{b})-\tilde{k}^{e}(\mathbf{b})-\tilde{k}^{p}(\mathbf{b})
\end{gathered}
$$

for determination of all unknown parameters b. From principle (22) using Eq. (5) we obtain

$$
\begin{array}{r}
\int_{\varepsilon_{1}^{*}}^{\varepsilon_{2}^{*}} \int_{V_{n}^{*}} \sigma^{*}: d\left(\varepsilon^{e *}+\varepsilon^{f *}\right) d V_{n}-\int_{V_{n}^{*}}\left(\psi_{2}^{*}-\psi_{1}^{*}\right) d V_{n}-\int_{V_{n}^{*}} k_{n}^{e *} d V_{n}<0= \\
\int_{\varepsilon_{1}}^{\varepsilon_{2}} \int_{V_{n}} \boldsymbol{\sigma}: d\left(\varepsilon^{e}+\boldsymbol{\varepsilon}^{f}\right) d V_{n}-\int_{V_{n}}\left(\psi_{2}-\psi_{1}\right) d V_{n}-\int_{V_{n}} k_{n}^{e} d V_{n}=0 .
\end{array}
$$

Corresponding principles for points of coherent and noncoherent interfaces, based on principle (24), are given in detail in $[4,5]$. Note, that our nonstrict conclusion after Eq. (19), that under prescribed horizontal displacement $u$ PT should start at minimal value $\sigma_{n}^{1 \rightarrow 2}$, i.e. an infinitesimal narrow layer, can be now strictly derived using the principle (24). Under the same BC the inclined layer can appear inside the half space, and an "optimal" inclination angle can be also found with the help of principle (24). The same is valid for determination of real shape of noncoherent nucleus in the second problem. These problems will be considered elsewhere.

\section{Concluding remarks}

In the paper the general thermodynamical approach for the description of PT in elastoplastic materials is suggested and two examples are considered. Application of the obtained results for the averaged description of $\mathrm{PT}$ (using volume fractions) can be based on a. method suggested in $[1,2,8]$ for obtaining of simple equations for stresses in each phase of multiphase materials.

Acknowledgment - The financial support of Alexander von Humboldt Foundation, as well as discussions with Prof. E. Stein are gratefully acknowledged.

\section{References}

[1] Levitas V.I., Papers of the Ukrainian SSR Academy of Sciences, 8 (1990) 41-46.

[2] Levitas V.I., Thermomechanics of phase transformations and inelastic deformations in microinhomogeneous materials (Naukova Dumka, Kiev,1992).

[3] Fischer F.D. et. al., Arch. Appl. Mech. 64 (1994) 54-85.

[4] Levitas V.I., Post-bifurcation behaviour in finite elastoplasticity. Applications to strain localization and phase transitions (Universität Hannover, Institut für Baumechanik und Numerische Mechanik, IBNM-Bericht 92/5, 1992).

[5] Levitas V.I., Int. J. Eng. Sci. (1995, in press).

[6] Levitas V.I., Mech. Res. Commun. 3 (1994) 273-280.

[7] Alexandrova M.M. et. al., Soviet. Solid State Phys. 30 (1988) 577-579.

[8] Levitas V.I. and Stein E., ZAMM 75 (1995) S199-S200. 